\title{
ISFM-Award pour un engagement exceptionnel
}

\author{
Werner Bauera, Raphael Stolz ${ }^{\mathrm{b}}, \mathrm{Nadja}^{\mathrm{a}}$ Jennic \\ ${ }^{\text {a }}$ Dr, président de I'ISFM \\ ${ }^{b} \mathrm{Dr}$, vice-président de I'ISFM \\ ${ }^{c}$ M. Sc., collaboratrice scientifique FMH/ISFM
}

Correspondance: Institut suisse pour la formation médicale postgraduée et continue ISFM FMH

Elfenstrasse 18

CH-3000 Berne 15

Tél. 0313591111

siwf[at]fmh.ch
Le succès de la première édition de l'ISFM-Award l'année dernière a conforté notre opinion selon laquelle la reconnaissance et la mise en valeur des formateurs revêtent tout autant d'importance que de bonnes bases structurelles et financières pour la formation postgraduée. Présentée comme un projet-pilote en 2014, la remise de l'ISFM-Award est aujourd'hui amenée à devenir un événement fixe dans l'agenda de l'ISFM.

La responsabilité que portent les médecins-cadres en matière de formation postgraduée constitue un des principes fondamentaux du transfert de connaissances et de compétences aux jeunes médecins. Pour les formateurs, faire preuve d'un engagement suffisant relève certainement de leur cahier des charges. En revanche, il ne va pas nécessairement de soi qu'ils se consacrent à leur tâche avec un enthousiasme supérieur à la moyenne et qu'ils transmettent avec passion leurs connaissances et leurs expériences alors que la charge administrative ne cesse d'augmenter. C'est pourquoi l'ISFM entend donner la possibilité aux jeunes médecins de témoigner leur reconnaissance aux formateurs qui se distinguent par leur charisme, leur compétence ou leur créativité à enseigner les compétences médicales.

\section{Nomination par les anciens médecins-assistant(e)s}

Pour être nommés à l'ISFM-Award, les médecins doivent participer activement à la formation médicale postgraduée. Il s'agit notamment de médecins-cadres qui s'engagent personnellement dans la formation et que les futurs spécialistes considèrent comme particulièrement compétents dans la transmission des connaissances et aptitudes. Pour nommer un confrère, il faut être médecin en formation postgraduée ou avoir obtenu un titre de spécialiste il y a moins d'un an. Pour qu'une nomination soit valable, elle doit être déposée conjointement par deux personnes et exprimer une reconnaissance personnelle pour la qualité de la for- mation dispensée par le formateur et pour son engagement. Afin qu'il ne résulte ni avantage ni conflit en raison du processus de nomination, vous ne pouvez nommer que les responsables de la formation postgraduée chez lesquels vous ne travaillez plus. Les noms des personnes qui ont déposé une nomination ne seront ni publiés, ni communiqués aux nominés. Aucun classement ne sera établi.

\section{Déposez votre nomination sans attendre!}

Pour nommer quelqu'un, nous vous invitons à rédiger une brève lettre de nomination (env. 1000 à $2000 \mathrm{ca}$ ractères) au format Word ou PDF. Celle-ci doit comporter les points suivants:

- Informations relatives à la personne nommée (nom, prénom, e-mail, discipline, fonction, établissement de formation postgraduée avec adresse).

- Informations concernant les deux personnes à l'origine de la nomination (nom, prénom, e-mail, disci-

\section{Nommez sans attendre \\ des responsables de la formation!}

L'ISFM-Award permet de récompenser des responsables de la formation postgraduée particulièrement engagés et compétents. Un ancien formateur vous a-t-il laissé une impression durable? Alors nommez-le pour I'ISFM-Award pour un engagement exceptionnel dans la formation postgraduée! Nous vous prions d'envoyer votre lettre de nomination sous forme électronique à l'adresse siwf[at]fmh.ch avec la mention "ISFMAward - pour un engagement exceptionnel dans la formation postgraduée».

Délai d'envoi: 9 août 2015.

Vous trouverez d'autres informations sur www.siwf.ch. Si vous avez des questions, adressez-vous à siwf[at]fmh.ch ou au numéro 0313591111.

Mention légale: suivant les circonstances, I'ISFM se réserve le droit de suspendre le projet AWARD ou de modifier les conditions de participation. Aucune correspondance ne sera tenue au sujet de I'ISFM-Award. Tout recours juridique est exclu. 


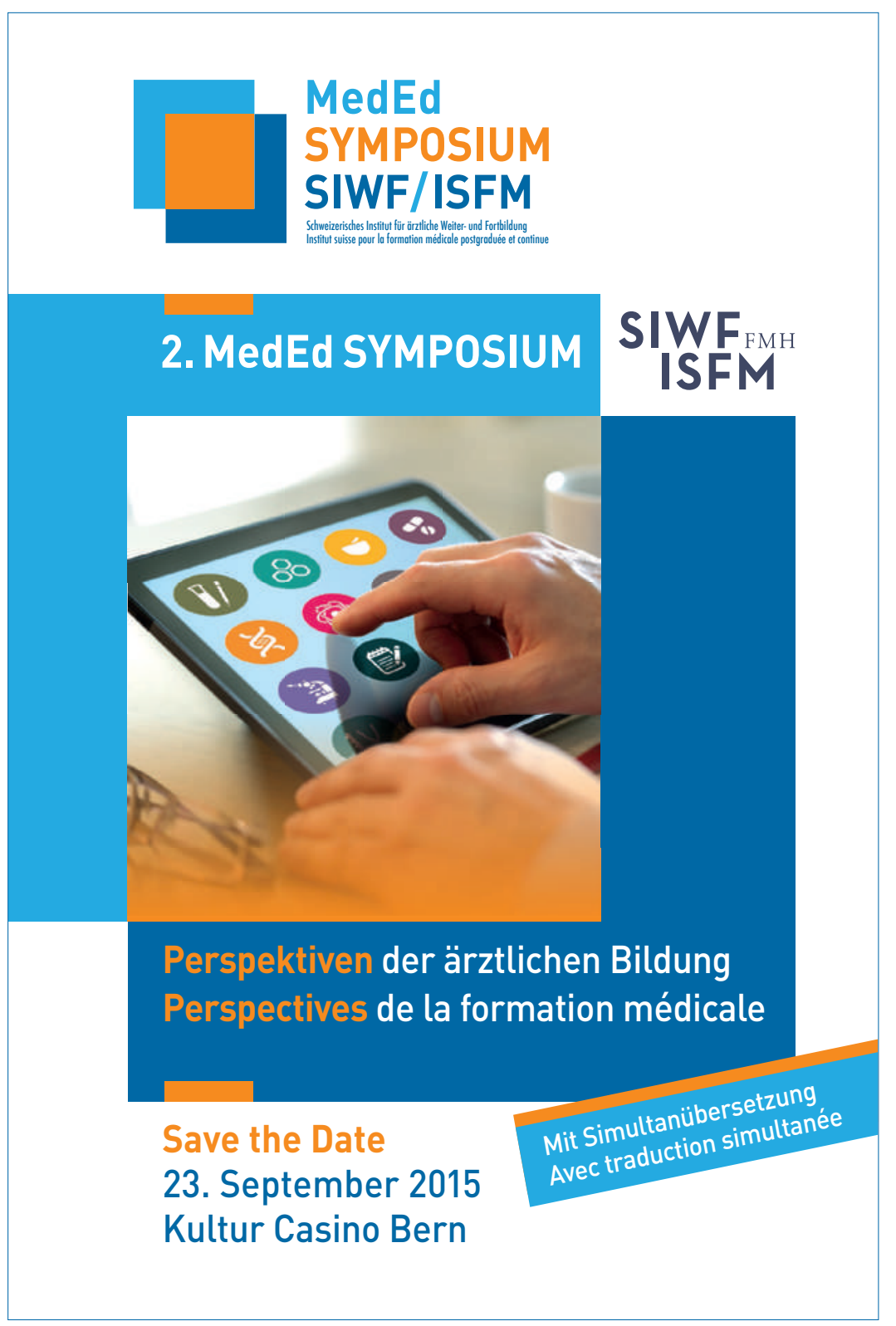

pline, dates de la période de formation postgraduée auprès de la personne nommée).

- Motifs de la nomination: citez au moins deux exemples concrets de situations lors desquelles la personne nommée vous est apparue comme particulièrement compétente, créative et engagée dans la formation postgraduée.

Présentée comme un projet-pilote en 2014, la remise de l'ISFM-Award est aujourd'hui amenée à devenir un événement fixe dans l'agenda de l'ISFM.

Vous pouvez envoyer votre lettre de nomination sous forme électronique jusqu'au 9 août 2015 à siwf[at] fmh.ch avec la mention "ISFM-Award pour un engagement exceptionnel dans la formation postgraduée». La direction de l'ISFM contrôlera si la nomination est correcte du point de vue formel et validera ensuite chaque nomination. Tous les médecins correctement nommés recevront un acte de reconnaissance et un cadeau en récompense de leur engagement dans la formation postgraduée. Ils seront cités nommément (après accord) sur le site web de l'ISFM (www.siwf.ch) et lors du MedEd-Symposium du 23 septembre 2015. 